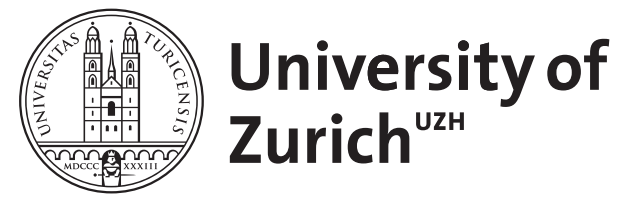
Archive

University of Zurich

University Library

Strickhofstrasse 39

CH-8057 Zurich

www.zora.uzh.ch

Year: 2013

\title{
Zum Gedenken an Prof. Dr. Dr. h.c. Urs Walter Schnyder
}

Burg, G ; French, L ; Hafner, J

DOI: https://doi.org/10.1007/s00105-012-2500-2

Posted at the Zurich Open Repository and Archive, University of Zurich

ZORA URL: https://doi.org/10.5167/uzh-156529

Journal Article

Published Version

Originally published at:

Burg, G; French, L; Hafner, J (2013). Zum Gedenken an Prof. Dr. Dr. h.c. Urs Walter Schnyder. Der Hautarzt, 64(1):65-66.

DOI: https://doi.org/10.1007/s00105-012-2500-2 
Hautarzt 2013 · 64:65-66

DOI 10.1007/s00105-012-2500-2

Online publiziert: 22. Dezember 2012

c) Springer-Verlag Berlin Heidelberg 2012

G. Burg ${ }^{1,2} \cdot$ L. French ${ }^{1} \cdot$ J. Hafner ${ }^{1}$

${ }^{1}$ Dermatologische Klinik, UniversitätsSpital Zürich, Zürich

${ }^{2}$ Maur/ZH

\section{Zum Gedenken an Prof. Dr. Dr. h.c. Urs Walter Schnyder}

\section{Februar 1923 bis 21. Oktober 2012}

In der Zeit, als sich die Dermatologie unter Bruno Bloch in Zürich zu etablieren begann, wurde Urs Walter Schnyder (- Abb. 1) am 7. Februar 1923 in Basel geboren. Er entstammte einer großbürgerlichen Familie und war der einzige Sohn von Sylvia Schnyder, der Tochter des Chemikers und Direktors der damaligen Sandoz in Basel, und von Walter Schnyder, Chefarzt der Augenklinik im Bürgerspital Solothurn in der Schweiz. Großvater und Urgroßvater waren Allgemeinärzte. Großonkel Édouard Guillaume war Physiker und hat 1920 den Nobelpreis für die Einführung des Urmeters erhalten.

\section{Werdegang}

Die Schulen besuchte Urs Schnyder in Solothurn, wo er die Matura mit Bestnoten abschloss. Auf einem Offiziersball in Basel lernte er Carmen, seine Frau, kennen. Drei Kinder, Christoph, Isabel und Andreas, entsprossen dieser glücklichen, langen Ehe.

Nach dem Medizinstudium in Bern, Genf und Zürich war Urs Schnyder 3 Jahre in der Pathologie tätig, zunächst in Genf und anschließend in Zürich. Seine wissenschaftlichen und klinischen Interessen lagen aber im Gebiet der Dermatologie, sodass er 1952 seine dermatologische Ausbildung bei Prof. Guido Miescher in Zürich begann. Nach Studienaufenthalten an der dermatologischen Klinik in München, am „Institut de Géné- tique Humaine“ der Universitäts-Augenklinik in Genf, am Hôpital Saint-Louis in Paris sowie an verschiedenen dermatologischen Kliniken und genetischen Instituten in den USA wurde er Oberarzt an der dermatologischen Klinik in Zürich bei Prof. Hans Storck.

Mit Beginn des Sommersemesters 1961 erhielt Urs Schnyder mit seiner Habilitationsschrift „Neurodermitis - Asthma - Rhinitis - eine genetisch-allergologische Studie“ die Venia legendi für Dermatologie und Venerologie an der Medizinischen Fakultät Zürich. Bereits 3 Jahre später wurde er zum Assistenzprofessor für Dermatologie mit besonderer Berücksichtigung der Humangenetik ernannt.

Im jugendlichen Alter von 42 Jahren erhielt Urs Schnyder 1965 den Ruf auf den renommierten Lehrstuhl für Dermatologie und Venerologie der Universitäts-Hautklinik Heidelberg. Dies war eine wichtige Zeit für ihn und für die Dermatologie. In dem von ihm verfassten Beitrag „Emeriti erinnern sich“ schreibt er: „Mit Freude habe ich ... im Frühsommer 1965 den Ruf ,über die Grenzen hinweg“ angenommen, nachdem das Kultusministerium den ... Einbau einer elektronenmikroskopischen Forschungsabteilung zugesichert hatte." In Heidelberg entfaltete Urs Schnyder eine rege Forschungstätigkeit zur Analyse von Erbkrankheiten - besonders Epidermolysen und Verhornungsstörungen - mithilfe der Lichtund Elektronenmikroskopie, biochemi- schen und molekularbiologischen Methoden. Es entstand das Institut für Ultrastrukturforschung der Haut als eine in die Hautklinik integrierte Abteilung, die seit 1975 von seiner engen Mitarbeiterin, Frau Prof. Anton-Lamprecht, geleitet wurde. In die Weltliteratur eingegangen sind insbesondere die Arbeiten zusammen mit Anton-Lamprecht und Hashimoto über verschiedene Ichthyose- und Epidermolysetypen. Einige der neu beschriebenen Varianten sind heute eponym mit dem $\mathrm{Na}$ -

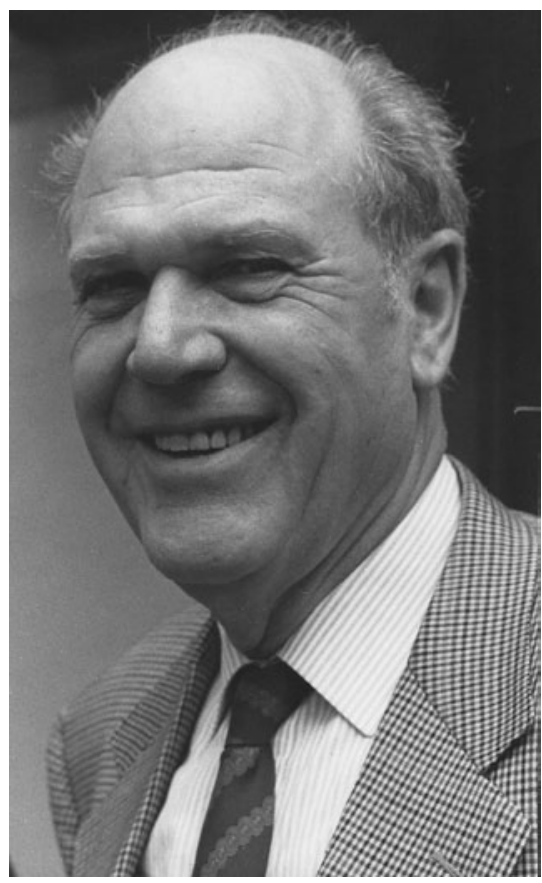

Abb. 1 A Prof. Dr. Urs Walter Schnyder 
men Schnyder verbunden. Besondere Erwähnung verdient auch seine Arbeit über die Palmoplantarkeratose Mal deMeleda, für die er eine Forschungsreise zur dalmatinischen Insel Mljet unternahm. Der zweite Schwerpunkt seiner Tätigkeiten betraf die Dermatohistopathologie. In die Heidelberger Zeit fällt die Herausgabe der „Histopathologie der Haut" in der Buchreihe "Spezielle pathologische Anatomie“ (Hrsg.: Doerr, Seifert, Ühlinger, 1973, 1979). Insgesamt war die akademische Bilanz der Heidelberger Zeit hervorragend.

Die Universität Zürich konnte stolz darauf sein, Professor Schnyder 1978 in die Schweiz zurückholen zu können. Hier hat er seine wissenschaftlichen Aktivitäten insbesondere auf dem Gebiet der Genodermatosen, aber auch der zur damaligen Zeit sich neu entwickelnden AidsProblematik fortgesetzt.

Im Jahr 1985 richtete Professor Schnyder die 34. Tagung der deutschsprachigen Dermatologie in Zürich aus. Das Thema der Tagung lautete „die Haut als Grenzorgan“. Es war eine der ersten medizinischen Tagungen überhaupt, bei der Patientenvorstellungen über Videokonferenz - damals Eidophor genannt - aus einem kleinen Ambulanzraum in ein großes Auditorium übertragen wurden.

Nachdem er sich bereits bei der Renovierung der Hautklinik Heidelberg baumeisterliche Verdienste erworben hatte, konnte Urs Schnyder zwischen 1985 und 1989 die 1924 von Bruno Bloch im neoklassizistischen Stil erbaute Hautklinik in Zürich zu einer der schönsten und modernsten Kliniken Europas umbauen.

\section{Auszeichnungen und Ämter}

Von seinen zahlreichen, hier nicht alle aufzuzählenden nationalen und internationalen Auszeichnungen und Ehrenmitgliedschaften sollen an dieser Stelle nur die folgenden besonderen Verdienste und Ämter von Urs Schnyder herausgestellt werden:

- In seine Zeit als Dekan der Heidelberger medizinischen Fakultät 1970/1971 fielen die unruhigen Post-68er-Jahre. Die hiermit verbundenen Probleme und Studentenunruhen hat er mit einer ausgewogenen Mischung von eindrucksvoller physischer Prä- senz und didaktischem und diplomatischem Geschick gemeistert.

- Von 1970 bis 1975 war er Senatsbeauftragter des Deutschen Krebsforschung-Zentrums (DKFZ) in Heidelberg.

- Er war Beauftragter des Rektors der Universität Heidelberg für die Universität Montpellier. Diese ehrwürdige medizinische Hochschule, die eine der ältesten in Europa ist, hat ihm 1982 für seine Verdienste die Ehrendoktorwürde verliehen.

- Prof. Schnyder war Mitglied und Senator für die Schweiz der Nationalen Akademie der Wissenschaften, Leopoldina.

- Nach seiner Emeritierung im Jahr 1991 übernahm Prof. Schnyder die Aufgaben eines Mittelsmannes der Universität für die interkantonalen Beziehungen mit seinem Geburtskanton Solothurn.

\section{Der Lehrer}

Als akademischer Lehrer hat es sich Prof. Schnyder nicht nehmen lassen, seine Vorlesungen stets selbst zu halten; und er füllte die Hörsäle sowohl in Heidelberg als auch in Zürich. Er hat das Fach Dermatologie und Venerologie national und international nachhaltig geprägt. Die Ausbildung der Assistenten, der Oberärzte und die akademischen Karrieren seiner Mitarbeiter waren ihm sehr wichtig. Die Studierenden und die Kolleginnen und Kollegen, die das große Glück hatten, ihre Weiterbildung bei Professor Schnyder absolvieren zu können, wurden nicht nur fachlich, sondern auch persönlich von ihm geprägt. Aus seiner Schule kamen die Lehrstuhlinhaber und Klinikleiter von Mannheim, Professor Jung, von Lausanne, Professor Pannizon, von Freiburg im Breisgau, Professor Schöpf und Frau Professor Bruckner, sowie der Leiter der Zürcher Städtischen Poliklinik, Prof. Eichmann, und der Leiter der Dermatologischen Klinik in Karlsruhe, Prof. Gloor.

\section{Der Privatmann}

Seine private Liebe zur Kunst äußerte sich nicht zuletzt auch beruflich in der Unterstützung der von Frau Elsbeth Stoiber und heute von Dr. Michael Geiges geleiteten Moulagensammlung der Klinik und der Universität.

Prof. Schnyder war ein hervorragender Wissenschaftler, ein didaktisch begeisternder Lehrer, ein strahlendes Vorbild mit natürlicher Autorität, ein großartiger Mensch mit Charisma und ein fürsorglicher Familienvater, dem seine liebe Frau Carmen stets unterstützend zur Seite stand. Er war ein Mäzen und ein großzügiger Sponsor, der unter anderem durch eine nach ihm benannte Stiftung für Genodermatosen die Forschung in diesem wichtigen Gebiet in der Schweiz neu belebt hat.

Nachdem er das Glück hatte, seine teilweise in Übersee beheimatete Familie in den letzten Tagen seines erfüllten Lebens um sich zu versammeln, ist Urs Walter Schnyder am 21. Oktober 2012 sanft entschlafen. Nach Angaben seiner Familie war eines seiner letzten Worte „wunderbar", und ein wunderbares Leben hat er tatsächlich im Privaten wie im Beruflichen gehabt.

Ein wunderbarer Mensch ist von uns gegangen. Wir werden ihn nicht verlieren, wenn wir ihn so in Erinnerung behalten, wie er war.

Prof. Günter Burg,

ehemaliger Klinikdirektor der Dermatologischen Klinik, UniversitätsSpital

Zürich

Prof. Lars French,

Klinikdirektor der Dermatologischen

Klinik, UniversitätsSpital Zürich

Prof. Jürg Hafner,

Präsident der SGDV, Leitender Arzt der

Dermatologischen Klinik, UniversitätsSpital Zürich

\section{Korrespondenzadresse}

Prof. Dr. G. Burg

Haldenstr. 14, 8124 Maur/ZH

Schweiz

g.burg@access.uzh.ch 